\title{
Etre les artisans de notre avenir
}

\author{
Jürg Schlup \\ Dr méd., président de la FMH
}

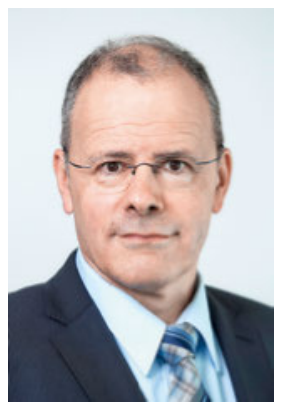

Le début de l'année, c'est aussi le moment de se demander de quoi sera fait l'avenir, de passer en revue ce qui s'est bien passé et ce que nous pourrions améliorer. Intuitivement, nous savons que l'avenir est l'enfant du présent [1]: si nous ne pouvons pas le prédire, nous pouvons souvent l'influencer.

Ce qui vaut pour notre avenir s'applique aussi à l'avenir de notre profession. En matière de santé publique, plusieurs interventions parlementaires déjà en cours vont marquer l'année 2018 et, peut-être aussi à long terme, notre avenir professionnel et celui de toute la profession. Alors que l'attention de beaucoup d'entre nous se focalise sur les effets déplorables de la deuxième intervention tarifaire et que la frustration compréhensible - atteint son paroxysme, le Parlement envisage d'enterrer définitivement l'autonomie tarifaire pour la remplacer par un tarif étatique durable. Et

\section{Le corps médical a du succès lorsqu'il est uni et propose des solutions.}

ce n'est pas tout: plusieurs propositions de régulation encore plus néfastes sont en route, justifiées par les polémiques sur la maîtrise des coûts. La mise en place de listes d'opérations ambulatoires, prévues sans introduire simultanément le financement uniforme des prestations ambulatoires et hospitalières, augmentera forcément les primes. Cela renforcera les partisans du budget global pour les prestations financées par l'AOS, tout en préparant le terrain pour le rationnement des soins. Mais aussi la clause du besoin revue et corrigée, les interventions parlementaires en vue de supprimer le libre choix du médecin et les exigences de qualité couplées à des sanctions ont le potentiel de changer profondément et négativement toute la prise en charge médicale de notre pays.

En 2018, nous serons donc confrontés à plusieurs projets dont la mise en œuvre risque de pénaliser bien plus que les patients. L'exercice libéral de la médecine, tel que nous le connaissons aujourd'hui, pourrait n'être plus qu'un lointain souvenir. Si des objectifs économiques, issus des politiques, des caisses-maladie ou des employeurs, se dressent entre les patients et nous, et dirigent notre manière d'exercer, ils se demande-

ront ce qui importe pour nous: leur état de santé ou les dispositions budgétaires? L'absence d'indépendance remettrait en question notre crédibilité et l'indispensable relation de confiance avec les patients. Nous ne serions que les administrateurs de patients pour une médecine commercialisée.

Mark Twain aurait un jour déclaré, ce qui nous parle à tous: "Si l'avenir m'intéresse, c'est parce que je vais y passer le reste de ma vie» [2]. L'effet contre-productif de certaines décisions peut se révéler par la suite, comme le rejet de notre proposition de révision du tarif lors de la votation générale de juin 2016, qui a ouvert la porte pour la deuxième intervention tarifaire et qui est utilisé aujourd'hui pour légitimer les jalons posés pour un tarif étatique. Du même coup, nous avons réduit notre capacité de réagir dans une phase politique décisive.

Dans ce contexte, que pouvons-nous améliorer aujourd'hui pour nous assurer le meilleur avenir possible? Nous devons nous unir, et ce en tant que partie de la solution! Le corps médical a généralement du succès lorsqu'il n'est pas divisé et tend la main à ses partenaires pour trouver des solutions. Il faudra certes argumenter de manière constructive mais aussi proposer des solutions face aux défis à venir. Cela signifie également présenter un tarif commun, même si cela demande un effort, car la deuxième intervention tarifaire doit rester l'ultime intrusion dans une révision tarifaire douloureuse et ne pas devenir la première

\section{Notre liberté aura un avenir si nous sommes à la hauteur de nos responsabilités.}

phase d'un tarif étatique inadmissible. En tant que membres d'une profession libérale, nous avons la responsabilité de travailler de manière constructive à des solutions sans perdre de vue la réalité politique. Donner un avenir à notre liberté dépend aussi de savoir si nous sommes à la hauteur de nos responsabilités.

\section{Références}

1 «Zukunft ist ein Kind der Gegenwart.» Christoph August Tiedge (1752-1841), poète allemand.

2 Mark Twain (1835-1910), de son vrai nom Samuel Langhorne Clemens, écrivain, essayiste et humoriste américain. 